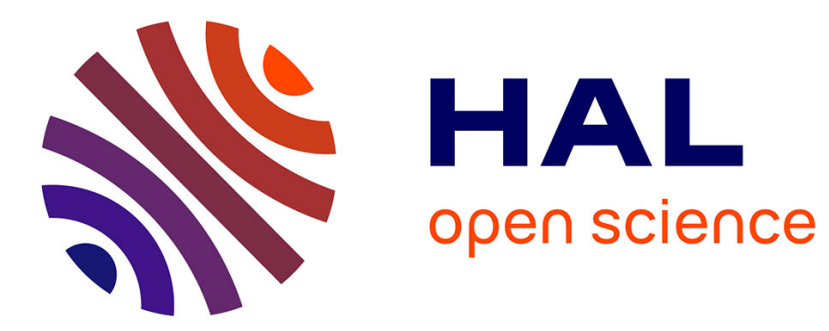

\title{
Robust backstepping for the Euler approximate model of sampled-data strict-feedback systems
}

\author{
R. Postoyan, Tarek Ahmed-Ali, Françoise Lamnabhi-Lagarrigue
}

\section{To cite this version:}

R. Postoyan, Tarek Ahmed-Ali, Françoise Lamnabhi-Lagarrigue. Robust backstepping for the Euler approximate model of sampled-data strict-feedback systems. Automatica, 2009, 45 (9), pp.2164-2168. 10.1016/j.automatica.2009.05.001 . hal-01058825

\section{HAL Id: hal-01058825 \\ https://hal.science/hal-01058825}

Submitted on 28 Aug 2014

HAL is a multi-disciplinary open access archive for the deposit and dissemination of scientific research documents, whether they are published or not. The documents may come from teaching and research institutions in France or abroad, or from public or private research centers.
L'archive ouverte pluridisciplinaire $\mathbf{H A L}$, est destinée au dépôt et à la diffusion de documents scientifiques de niveau recherche, publiés ou non, émanant des établissements d'enseignement et de recherche français ou étrangers, des laboratoires publics ou privés. 


\title{
Robust backstepping for the Euler approximate model of sampled-data strict-feedback systems ${ }^{\text {th }}$
}

\author{
Romain Postoyan $^{\mathrm{a}, *}$, Tarek Ahmed-Ali $^{\mathrm{b}}$, Françoise Lamnabhi-Lagarrigue ${ }^{\mathrm{c}}$ \\ a Univ Paris-Sud, LSS-CNRS Supélec, 3 rue Joliot Curie, 91192 Gif-sur-Yvette, France \\ ${ }^{\mathrm{b}}$ Univ Caen Basse Normandie, GREYC-CNRS, 6 boulevard du Maréchal Juin, 14050 Caen Cedex 9, France \\ ${ }^{c}$ EECI, LSS-CNRS Supélec, 3 rue Joliot Curie, 91192 Gif-sur-Yvette, France
}

\begin{abstract}
A B S T R A C T
Stabilization of uncertain sampled-data strict-feedback systems is addressed. The stability study is carried out on the Euler approximation of the exact discretized model of the plant. Firstly, a class of state-feedback controllers is developed that guarantees an input-to-state stability property for the closed-loop system. Additionally, assuming some hypotheses on the uncertain terms hold, a practical asymptotic stability property is ensured by designing an appropriate class of controllers.
\end{abstract}

Keywords:

Sampled-data systems

Backstepping

Robust stabilization

\section{Introduction}

Among the recent literature on nonlinear sampled-data systems, few papers provide constructive stabilization results. In Nešić and Grüne (2005), a class of trajectory based controllers (see Grüne, Worthmann, and Nešić (2007) for a deeper study of this type of stabilizer) and sufficient conditions for the design of 'high gain' controllers are given that guarantee some practical stability properties, under some conditions. For an extension of the "high gain' type stabilizers to the adaptive case, see Postoyan, Ahmed-Ali, Burlion, and Lamnabhi-Lagarrigue (2008). In Burlion (2007), feedforward techniques have been developed for high order approximations. Backstepping control for the Euler approximate model of a class of nonlinear sampled-data systems has been investigated in Nešić and Teel (2006). Although not proved, simulations of several examples show that designed discrete-time controllers may notably enlarge the region of attraction compared to the emulation of the continuous-time control law.

On the other hand, the problem of stabilization of nonlinear sampled-data systems affected by uncertainties and/or perturbations has not received much attention. For results on ISS and/or

\footnotetext{
This paper was not presented at any IFAC meeting. This paper was recommended for publication in revised form by Associate Editor Mingqing Xiao under the direction of Editor André L. Tits.

* Corresponding author. Tel.: +330 169851772; fax: +330169851765.

E-mail addresses: postoyan@lss.supelec.fr (R. Postoyan), tarek.ahmed-ali@greyc.ensicaen.fr (T. Ahmed-Ali), lamnabhi@lss.supelec.fr (F. Lamnabhi-Lagarrigue).
}

IOSS (input-to-(output-)state Stability) properties for parameterized discrete-time systems, see Laila and Nešić (2002a), Laila and Nešić (2002b) and Laila and Nešić (2003). In Kellett, Shim, and Teel (2004), robustness properties of emulated controllers (possibly discontinuous) have been investigated but under severe conditions, notably on the sampling period. In Laila and Astolfi (2005), semiglobal practical input-to-state stability (SP-ISS) for uncertain time-varying parameterized discrete-time systems is addressed and the design of controllers for nonholonomic systems in power form is realized. For results on singularly perturbed sampled-data systems, see Barbot, Djemai, Monaco, and Normand-Cyrot (1996) and references therein.

In this paper, stabilization of sampled-data systems in strictfeedback form with single input is investigated. Based on the framework proposed in Nešić and Teel (2004), the stability study is carried out on the Euler approximation of the exact discretized system. Firstly, a class of controllers is designed that ensures the SP-ISS of the exact discrete-time model. Afterwards, attention is focused on the case where the uncertain terms are known to satisfy some hypotheses. A class of controllers is then derived that ensures a semiglobal practical asymptotic stability (SP-AS) objective. Contrary to (Kellett et al., 2004), where the stabilization via backstepping techniques of a class of systems using an emulated controller has been done, controllers are here synthesized on an approximation of the exact discretized system. In that way, the obtained controllers are 'redesigned' similarly to Monaco and Normand-Cyrot (2001), Nešić and Grüne (2005) and Nešić and Teel (2006). Moreover, the type of perturbations/uncertainties is larger 
here than those allowable in Kellett et al. (2004). Note that this work can be considered as the robust version of the results in Nešić and Teel (2006).

\subsection{Nomenclature and mathematical framework}

First some mathematical notations are introduced. Let $\mathbb{R}=$ $(-\infty, \infty), \mathbb{R}_{>0}=(0, \infty), \mathbb{R}_{\geq 0}=[0, \infty), \mathbb{N}_{0}=\mathbb{N} \cup\{0\}$ and let the Euclidean norm be denoted by $|\cdot|$. In all this study, the initial time is chosen to be zero (without loss of generality). A function $\gamma: \mathbb{R}_{\geq 0} \longrightarrow \mathbb{R}_{\geq 0}$ is called $\mathcal{K}$ if it is continuous, zero at zero, strictly increasing and of class $\mathcal{K}_{\infty}$ if it is unbounded. A continuous function $\gamma: \mathbb{R}_{\geq 0} \times \mathbb{R}_{\geq 0} \longrightarrow \mathbb{R}_{\geq 0}$ is of class $\mathcal{K} \mathcal{L}$ if, for each $t \in \mathbb{R}_{>0}, \gamma(\cdot, t)$ is of class $\mathcal{K}$, and, for each $s \in \mathbb{R}_{>0}, \gamma(s, \cdot)$ is decreasing to zero. For the sake of simplicity, the notations $z$ and $z^{+}$, where $z$ is a time-dependent variable, will be used to denote $z(k T), z((k+1) T)$, respectively, where $k \in \mathbb{N}_{0}, T \in \mathbb{R}_{>0}$. For a function $d: \mathbb{R}_{\geq 0} \rightarrow \mathbb{R}^{n}, d[k T]$ denotes $\{d(t): t \in[k T,(k+1) T)\}$, $k \in \mathbb{N}_{0}, n \in \mathbb{N}, T \in \mathbb{R}_{>0}$. It is said that $d \in \mathscr{L}_{\infty}$ if $d$ is Lebesgue measurable and there exists $r \in \mathbb{R}_{\geq 0}$ such that $\|d\|_{\infty}=$ $\sup _{\tau \in \mathbb{R}_{>0}}|d(\tau)| \leq r$ and $\left\|d_{f}\right\|_{\infty}$ denotes $\sup _{\tau \in[k T,(k+1) T)}|d(\tau)|, k \in$ $\mathbb{N}_{0}, T \in \mathbb{R}_{>0}$.

Without loss of generality, the sampling period, $T \in \mathbb{R}_{>0}$, is always assumed to be $T<1$ (this can be achieved by doing a rescale transformation of the sampling period if necessary).

\subsection{Definitions}

Consider the nonlinear systems described by

$\dot{x}(t)=f(x(t), u(t), d(t))$

where $x \in \mathbb{R}^{n}$ is the state vector, $u \in \mathbb{R}^{m}$ the input and $d \in \mathbb{R}^{m}$ the exogenous disturbance. The function $f$ is locally Lipschitz with $f(0,0,0)=0$. Function $d$ is Lebesgue measurable and the control $u$ is sampled at a given constant period $T \in \mathbb{R}_{>0}$. A sample-and-hold device is considered. The exact discretized system of (1) is given by, over $[k T,(k+1) T)$ for $k \in \mathbb{N}_{0}$,

$x((k+1) T)=F_{T}(x(k T), u(k T), d[k T])$.

Definition 1 (Laila $\mathcal{E}$ Nešić, 2002a). System (2) is said to be SPISS (semiglobally practically input-to-state stable) if there exists $\beta \in \mathcal{K} \mathcal{L}$ and $\gamma \in \mathcal{K}$, such that, for any $\Delta_{x}, \Delta_{d}, \delta \in \mathbb{R}_{>0}$, there exists $T^{*} \in \mathbb{R}_{>0}$ such that solutions of system (2) satisfy, for all $k \in \mathbb{N}_{0}, T \in\left(0, T^{*}\right),\left|x_{0}\right| \leq \Delta_{x}$ and $d \in \mathcal{L}_{\infty}$ with $\|d\|_{\infty} \leq \Delta_{d}$ :

$\left|x\left(k, x_{0}, d\right)\right| \leq \beta\left(\left|x_{0}\right|, k\right)+\gamma\left(\|d\|_{\infty}\right)+\delta$.

In the case where $\left|x\left(k, x_{0}, d\right)\right| \leq \beta\left(\left|x_{0}\right|, k\right)+\delta$, system (2) is said to be SP-AS (semiglobally practically asymptotically stable).

Definition 2 (Laila Er Nešić, 2002a). System (2) is Lyapunov SP-ISS if there exists a parameterized family of functions $V_{T}: \mathbb{R}^{n} \rightarrow \mathbb{R}_{\geq 0}$ such that there exist $\alpha_{i} \in \mathcal{K}_{\infty}, i \in\{1,3\}$, and $\gamma \in \mathcal{K}$, and for any $\Delta_{x}, \Delta_{d}, \delta_{1}, \delta_{2} \in \mathbb{R}_{>0}$ there exist $T^{*}, L \in \mathbb{R}_{>0}$ such that, for all $T \in\left(0, T^{*}\right),|x| \leq \Delta_{x}$ and all $d \in \mathcal{L}_{\infty}$ with $\|d\|_{\infty} \leq \Delta_{d}$, the following holds:

$$
\begin{aligned}
& \alpha_{1}(|x|) \leq V_{T}(x) \leq \alpha_{2}(|x|), \\
& \frac{1}{T}\left[V_{T}\left(F_{T}(x(k T), u(k T), d[k T])\right)-V_{T}(x)\right] \leq-\alpha_{3}(|x|) \\
& \quad+\gamma\left(\left\|d_{f}\right\|_{\infty}\right)+\delta_{1},
\end{aligned}
$$

and, for all $x_{1}, x_{2}, z$ with $\left|\left[x_{1}^{\mathrm{T}}, z^{\mathrm{T}}\right]^{\mathrm{T}}\right|,\left|\left[x_{2}^{\mathrm{T}}, z^{\mathrm{T}}\right]^{\mathrm{T}}\right| \in\left[\delta_{2}, \Delta_{x}\right]$ and all $T \in\left(0, T^{*}\right),\left|V_{T}\left(x_{1}, z\right)-V_{T}\left(x_{2}, z\right)\right| \leq L\left|x_{1}-x_{2}\right|$. Moreover, if $d=0$, system $(2)$ is said to be Lyapunov $\mathbf{S P}$-AS. The pair $\left(u, V_{T}\right)$ is called an SP-IS stabilizing (SP-AS) pair.
In the general case where the exogenous signal $d$ is only Lebesgue measurable, the following Euler-like approximation of the exact discretized system (2) is considered, for $k \in \mathbb{N}_{0}$ :

$x((k+1) T)=x(k T)+\int_{k T}^{(k+1) T} f(x(k T), u(k T), d(s)) \mathrm{d} s$.

When $d$ is known to be continuously differentiable (like in Section 3), the Euler approximate model can take the classical form, for $k \in \mathbb{N}_{0}$ :

$x((k+1) T)=x(k T)+T f(x(k T), u(k T), d(k T))$.

In both cases, models (6) and (7) are a strong consistent approximation of (2) (see Laila and Nešić (2002a)). Hence stability properties for (2) can deduced from the stability analysis of (6) (or (7)) according to the following theorem, which is a direct consequence of Theorem 3.2 in Laila and Nešić (2002a).

Theorem 3. If system (6) (or (7)) is Lyapunov SP-ISS (SP-AS) and if the input $u$ is uniformly locally bounded, then the exact discretized system (2) is SP-ISS (SP-AS).

Stability properties of the sampled-data system (1) can then be deduced from those of the exact discretized system under mild conditions (Nešić, Teel, \& Sontag, 1999).

\subsection{Problem statement}

The purpose of this study is to propose control laws that guarantee some semiglobal practical stability properties for strictfeedback systems:

$\dot{\eta}=f(\eta)+g(\eta) \xi+d_{1}$

$\dot{\xi}=u+d_{2}$,

where $x=\left[\eta^{\mathrm{T}}, \xi\right]^{\mathrm{T}}$, with $\eta \in \mathbb{R}^{n}$ and $\xi \in \mathbb{R}$, is the state vector, and $u \in \mathbb{R}$ the control input that is sampled and held at a given constant period $T \in \mathbb{R}_{>0}$. The vector fields $f \in C^{1}\left(\mathbb{R}^{n}, \mathbb{R}^{n}\right)$ and $g \in$ $C^{1}\left(\mathbb{R}^{n}, \mathbb{R}\right)$ are supposed to be known and $f(0)=0$, and the signal $d=\left[d_{1}^{\mathrm{T}}, d_{2}\right]^{\mathrm{T}} \in \mathcal{L}_{\infty}$ is unknown and models the uncertainties or perturbations acting on the system.

It will be shown that the obtained controllers are 'redesigned' compared to the emulation of the continuous-time control law (see Monaco and Normand-Cyrot (2001), Nešić and Grüne (2005) and Nešić and Teel (2006) for other redesigned controllers for nonlinear sampled-data systems).

\section{Semiglobal practical input-to-state stabilization}

As mentioned in the Section 1, the Euler approximate model of the sampled-data system is considered:

$\eta^{+}=\eta+T(f(\eta)+g(\eta) \xi)+\tilde{d}_{1}$

$\xi^{+}=\xi+T u+\tilde{d}_{2}$,

where $\tilde{d}_{i}=\int_{k T}^{(k+1) T} d_{i}(s) \mathrm{d} s, i \in\{1,2\}$. Before giving the main result of this section, the following hypothesis is stated.

Hypothesis 4. There exist $\hat{T} \in \mathbb{R}_{>0}$ and an SP-ISS pair $\left(\bar{\xi}_{T}, W_{T}\right)$ defined for each $T \in(0, \hat{T})$ for subsystem (10), with $\xi \in \mathbb{R}$ regarded as its control. Suppose also that:

(1) $\bar{\xi}_{T}$ and $W_{T}$ are twice differentiable for any $T \in(0, \hat{T})$;

(2) there exists $\tilde{\phi} \in \mathcal{K}_{\infty}$ such that $\left|\bar{\xi}_{T}(\eta)\right| \leq \tilde{\phi}(|\eta|)$, for all $\eta \in \mathbb{R}^{n}$, $T \in(0, \hat{T})$;

(3) for any $\tilde{\Delta}>0$ there exists a pair of strictly positive numbers $\left(\tilde{T}, \tilde{M}_{1}\right)$ such that each $T \in(0, \tilde{T})$ and $|\eta| \leq \tilde{\Delta}$, $\max \left\{\left|\frac{\partial W_{T}}{\partial \eta}\right|\right.$, $\left.\left|\frac{\partial \bar{\xi}_{T}}{\partial \eta}\right|,\left|\frac{\partial^{2} \bar{\xi}_{T}}{\partial \eta^{2}}\right|,\left|\frac{\partial^{2} W_{T}}{\partial \eta^{2}}\right|,\left.\left|\frac{\partial}{\partial \eta}\right| \frac{\partial \bar{\xi}_{T}}{\partial \eta}\right|^{2} \mid\right\} \leq \tilde{M}_{1}$. 
The proof of the following theorem is omitted due to space limitations. However, it goes along the same lines as the proof of Theorem 12.

Theorem 5. Assuming Hypothesis 4 holds, defining, with $c \in \mathbb{R}_{>0}$,

$$
\begin{aligned}
& u_{T}(x)=-(c+1-c T)\left(\xi-\bar{\xi}_{T}(\eta)\right)+\frac{\bar{\xi}_{T}\left(\eta_{0}^{+}\right)-\bar{\xi}_{T}(\eta)}{T} \\
& -\left(\frac{\partial W}{\partial \eta}\left(\bar{\eta}_{0}^{+}\right)\right)^{\mathrm{T}} g(\eta)-(1-c T)\left(\xi-\bar{\xi}_{T}(\eta)\right)\left|\frac{\partial \bar{\xi}_{T}}{\partial \eta}\left(\eta_{0}^{+}\right)\right|^{2},
\end{aligned}
$$

with $\bar{\eta}_{0}^{+}=\eta+T\left[f(\eta)+g(\eta) \bar{\xi}_{T}\right], \eta_{0}^{+}=\eta+T[f(\eta)+g(\eta) \xi]$, system (10)-(12) is SP-ISS, and so is the exact discretized system of (8) and (9) controlled by (12).

Remark 6. The controllers (12) are of the form $u_{T}=u_{\text {cont }}+$ $T u_{d t}$, where $u_{\text {cont }}$ corresponds to the emulation of the continuoustime controller and $u_{d t}$ is an additional component that may allow enlarging the domain of attraction and increasing the speed convergence compared to the straight emulation, as can be seen in an example in Section 5.

\section{Semiglobal practical asymptotic stabilization}

In this section, some information on the uncertain terms is supposed to be available.

Hypothesis 7. (i) $d_{1} \in C^{1}\left(\left[t_{0}, \infty\right) \times \mathbb{R}^{n+1}, \mathbb{R}^{n}\right)$ and $d_{2} \in C^{1}$ $\left(\left[t_{0}, \infty\right) \times \mathbb{R}^{n+1}, \mathbb{R}\right)$.

(ii) There exist known functions $\rho_{1} \in C^{1}\left(\mathbb{R}^{n}, \mathbb{R}_{\geq 0}\right)$ with $\rho_{1}(0)=0$, $\rho_{2} \in C^{1}\left(\mathbb{R}^{n+1}, \mathbb{R}_{>0}\right)$ such that, for all $(t, x) \in\left[t_{0}, \infty\right) \times \mathbb{R}^{n+1}$ : $\left|d_{1}(t, x)\right| \leq \rho_{1}(\eta)$ and $\left|d_{2}(t, x)\right| \leq \rho_{2}(x)$.

Remark 8. This type of hypothesis is standard when dealing with perturbed strict-feedback systems (Freeman \& Kokotović, 1993).

Since condition (i) in Hypothesis 7 will be assumed to hold, the following approximate discrete-time model of the exact discretized system of (8) and (9) is considered, as mentioned in Section 1.2:

$\eta^{+}=\eta+T\left(f(\eta)+g(\eta) \xi+d_{1}\right)$

$\xi^{+}=\xi+T\left(u+d_{2}\right)$.

The following functions will be useful in what follows.

Definition 9. For any $\varepsilon, T \in \mathbb{R}_{>0}, n \in \mathbb{N}$, the function sat $\operatorname{sen}_{T \varepsilon, n}$ : $\mathbb{R}^{n} \rightarrow \mathbb{R}^{n}$ is defined as, for $z=\left[z_{1}, \ldots, z_{n}\right]^{\mathrm{T}} \in \mathbb{R}^{n}$, sat ${ }_{T \varepsilon, n}(z)=$ $\left[\text { sãt }_{T \varepsilon, 1}\left(z_{1}\right), \ldots, \text { sãt }_{T \varepsilon, 1}\left(z_{n}\right)\right]^{\mathrm{T}}$ with

$\operatorname{sant}_{T \varepsilon, 1}\left(z_{i}\right)= \begin{cases}\operatorname{sign}\left(z_{i}\right) & \text { if }\left|z_{i}\right| \geq \frac{T \varepsilon}{n} \\ p\left(z_{i}\right) & \text { otherwise }\end{cases}$

where $p: \mathbb{R} \mapsto \mathbb{R}, p(0)=0$ and $|p| \leq 1$ over $\left[-\frac{T \varepsilon}{n}, \frac{T \varepsilon}{n}\right], y p(y) \geq 0$ for $y \in\left[-\frac{T \varepsilon}{n}, \frac{T \varepsilon}{n}\right]$, is such that function sat ${ }_{T \varepsilon, n}$ is $C^{1}$ over $\mathbb{R}^{n}$.

Remark 10. There exist an infinite number of functions $p$ satisfying Definition 9 (see in Burlion (2007) and Freeman and Kokotović (1993) for examples).

Hypothesis 11. There exist $\hat{T} \in \mathbb{R}_{>0}$ and an SP-AS pair $\left(\bar{\xi}_{T}, W_{T}\right)$ defined for each $T \in(0, \hat{T})$ for subsystem (10), with $\xi \in \mathbb{R}$ regarded as its control. Suppose also that:

(1) $\bar{\xi}_{T}$ and $W_{T}$ are twice differentiable for any $T \in(0, \hat{T})$;

(2) there exists $\tilde{\phi} \in \mathcal{K}_{\infty}$ such that $\left|\bar{\xi}_{T}(\eta)\right| \leq \tilde{\phi}(|\eta|)$, for all $\eta \in \mathbb{R}^{n}, T \in(0, \hat{T})$;
(3) for any $\tilde{\Delta}>0$ there exists a pair of strictly positive numbers $\left(\tilde{T}, \tilde{M}_{1}\right)$ such that each $T \in(0, \tilde{T})$ and $|\eta| \leq \tilde{\Delta}, \max \left\{\left|\frac{\partial W_{T}}{\partial \eta}\right|\right.$, $\left.\left|\frac{\partial \bar{\xi}_{T}}{\partial \eta}\right|,\left|\frac{\partial^{2} \bar{\xi}_{T}}{\partial \eta^{2}}\right|,\left|\frac{\partial^{2} W}{\partial \eta^{2}}\right|\right\} \leq \tilde{M}_{1}$.

Theorem 12. Assuming Hypotheses 7 and 11 hold, defining, with $c \in \mathbb{R}_{>0}$,

$$
\begin{aligned}
u_{T}(x)= & -c\left(\xi-\bar{\xi}_{T}(\eta)\right)+\hat{d}_{2}+\frac{\bar{\xi}_{T}\left(\eta_{0}^{+}\right)-\bar{\xi}_{T}(\eta)}{T} \\
& -\left(\frac{\partial W}{\partial \eta}\left(\bar{\eta}_{0}^{+}\right)\right)^{\mathrm{T}} \mathrm{g}(\eta)-\left(\frac{\partial \bar{\xi}_{T}}{\partial \eta}\left(\eta_{0}^{+}\right)\right)^{\mathrm{T}} \hat{d}_{1},
\end{aligned}
$$

with $\bar{\eta}_{0}^{+}=\eta+T\left[f(\eta)+g(\eta) \bar{\xi}_{T}\right], \eta_{0}^{+}=\eta+T[f(\eta)+g(\eta) \xi]$, and $\hat{d}_{2}=-\rho_{2} s a t_{T \varepsilon, 1}\left(\left(\xi-\bar{\xi}_{T}(\eta)\right)\right)$ and $\hat{d}_{1}=\rho_{1} s a t_{T \varepsilon, n}\left\{\left(\xi-\bar{\xi}_{T}\right) \frac{\partial \bar{\xi}_{T}}{\partial \eta}\left(\eta_{0}^{+}\right)\right\}$, with $\varepsilon \in \mathbb{R}_{>0}$, then system (13)-(15) is SP-AS, and so is the exact discretized system of (8) and (9) controlled by (15).

Proof. Note that, for the sake of clarity, sat functions are called with no index in the proof. Let $\Delta, \delta, \varepsilon \in \mathbb{R}_{>0}, x=\left[\eta^{\mathrm{T}}, \xi\right]^{\mathrm{T}} \in$ $\mathbb{R}^{n+1}$ with $|x| \leq \Delta$. According to Hypothesis 11 , there exists $\hat{T} \in \mathbb{R}_{>0}$ such that condition (5) holds for $T \in(0, \hat{T})$ with $\frac{\delta}{2}$, considering system (13), when $\xi=\bar{\xi}_{T}$ as input. Let $\Delta_{1}=$ $\sup _{|x| \leq \Delta, T \in(0, \hat{T})} \max \left\{\left|\eta^{+}\right|,\left|\eta_{0}^{+}\right|,\left|\bar{\eta}_{0}^{+}\right|,\left|\bar{\eta}^{+}\right|\right\}$that is well defined since functions $f, g, \bar{\xi}_{T}, d_{1}$ are continuous. Let $\bar{\Delta}=\max \left\{\Delta, \Delta_{1}\right\}$ generate $\tilde{T}, \tilde{M}_{1}$ such that inequality 3 in Hypothesis 11 holds. Let $\tilde{M}=\sup _{|x| \leq \Delta, T \in(0, \hat{T})} \max \left\{\left|\xi-\bar{\xi}_{T}\right|,|f(\eta)+g(\eta) \xi|, \tilde{M}_{1},|g(\eta)|, \rho_{1}\right.$, $\left.\rho_{2}\right\}$, which is well defined since all the considered functions are continuous over the given compact set. The sampling period $\bar{T}$ is defined as $\bar{T}=\min \left\{\hat{T}, \tilde{T}, \frac{\delta}{2} \hat{M}^{-1}, \frac{1}{c}\right\}$, where $\hat{M}=10 \tilde{M}^{4}+(12+$ c) $\tilde{M}^{3}+4 \tilde{M}^{2}+4 \varepsilon \tilde{M}$. Let $T \in(0, \bar{T})$ and define the candidate Lyapunov function: $V_{T}(x)=W_{T}(\eta)+\frac{1}{2}\left(\xi-\bar{\xi}_{T}(\eta)\right)^{2}$. Condition (4) holds here; see Nešić and Teel (2006). Firstly, attention is focused on verifying that inequality (5) holds:

$$
\begin{aligned}
\Delta V_{T}= & W_{T}\left(\eta^{+}\right)-W_{T}(\eta)-\frac{1}{2}\left(\xi-\bar{\xi}_{T}(\eta)\right)^{2} \\
& +\frac{1}{2}\left(\xi+T u_{T}+T d_{2}-\bar{\xi}_{T}\left(\eta^{+}\right)\right)^{2} .
\end{aligned}
$$

It can be shown that, using the mean value theorem, with $\eta^{\diamond}=$ $\bar{\eta}^{+}+T \theta_{1} g(\eta)(\xi-\bar{\xi}(\eta))$, with $\theta_{1} \in(0,1)$,

$$
\begin{gathered}
W_{T}\left(\eta^{+}\right)-W_{T}(\eta)=W_{T}\left(\eta^{+}\right)-W_{T}\left(\bar{\eta}^{+}\right)+W_{T}\left(\bar{\eta}^{+}\right)-W_{T}(\eta) \\
=\left(W_{T}\left(\bar{\eta}^{+}\right)-W_{T}(\eta)\right)+\left(\frac{\partial W}{\partial \eta}\left(\eta^{\diamond}\right)\right)^{\mathrm{T}} \operatorname{Tg}(\eta)\left(\xi-\bar{\xi}_{T}(\eta)\right) .
\end{gathered}
$$

In view of (16), denoting $\Delta W_{T}=W_{T}\left(\bar{\eta}^{+}\right)-W_{T}(\eta)$,

$$
\begin{aligned}
\Delta V_{T} & =\Delta W_{T}+\left(\frac{\partial W}{\partial \eta}\left(\eta^{\diamond}\right)\right)^{\mathrm{T}} \operatorname{Tg}(\eta)\left(\xi-\bar{\xi}_{T}(\eta)\right) \\
& -\frac{1}{2}\left(\xi-\bar{\xi}_{T}(\eta)\right)^{2}+\frac{1}{2}\left(\xi+T u_{T}+T d_{2}-\bar{\xi}_{T}\left(\eta^{+}\right)\right)^{2} \\
= & \Delta W_{T}+\left(\frac{\partial W}{\partial \eta}\left(\eta^{\diamond}\right)\right)^{\mathrm{T}} \operatorname{Tg}(\eta)\left(\xi-\bar{\xi}_{T}(\eta)\right)-\frac{1}{2}\left(\xi-\bar{\xi}_{T}(\eta)\right)^{2} \\
& +\frac{1}{2}\left(\left(\xi-\bar{\xi}_{T}(\eta)\right)(1-c T)-T \frac{\partial \bar{\xi}_{T}}{\partial \eta}\left(\eta_{0}^{+}\right)^{\mathrm{T}} \hat{d}_{1}\right. \\
& \left.-T\left(\frac{\partial W}{\partial \eta}\left(\bar{\eta}_{0}^{+}\right)\right)^{\mathrm{T}} g(\eta)+T\left(\hat{d}_{2}+d_{2}\right)+\bar{\xi}_{T}\left(\eta_{0}^{+}\right)-\bar{\xi}_{T}\left(\eta^{+}\right)\right) \\
= & \Delta W_{T}+\left(\frac{\partial W}{\partial \eta}\left(\eta^{\diamond}\right)\right)^{\mathrm{T}} \operatorname{Tg}(\eta)\left(\xi-\bar{\xi}_{T}(\eta)\right)-c T\left(\xi-\bar{\xi}_{T}(\eta)\right)^{2} \\
& +c^{2} \frac{T^{2}}{2}\left(\xi-\bar{\xi}_{T}(\eta)\right)^{2}+(1-c T)\left(\xi-\bar{\xi}_{T}(\eta)\right) \Lambda+\frac{1}{2} \Lambda^{2},
\end{aligned}
$$


with

$$
\begin{aligned}
\Lambda= & \bar{\xi}_{T}\left(\eta_{0}^{+}\right)-\bar{\xi}_{T}\left(\eta^{+}\right)+T\left(-\left(\frac{\partial \bar{\xi}_{T}}{\partial \eta}\left(\eta_{0}^{+}\right)\right)^{\mathrm{T}} \hat{d}_{1}\right. \\
& \left.-\left(\frac{\partial W}{\partial \eta}\left(\bar{\eta}_{0}^{+}\right)\right)^{\mathrm{T}} g(\eta)+\hat{d}_{2}+d_{2}\right) .
\end{aligned}
$$

Thanks to the use of the mean value theorem,

$$
\begin{aligned}
- & T(1-c T)\left(\xi-\bar{\xi}_{T}(\eta)\right)\left(\frac{\partial W}{\partial \eta}\left(\bar{\eta}_{0}^{+}\right)\right)^{\mathrm{T}} g(\eta) \\
& +\left(\frac{\partial W}{\partial \eta}\left(\eta^{\diamond}\right)\right)^{\mathrm{T}} \operatorname{Tg}(\eta)\left(\xi-\bar{\xi}_{T}(\eta)\right) \\
\leq & T\left(\xi-\bar{\xi}_{T}(\eta)\right)\left(\frac{\partial W}{\partial \eta}\left(\eta^{\diamond}\right)-\frac{\partial W}{\partial \eta}\left(\bar{\eta}_{0}^{+}\right)\right)^{\mathrm{T}} g(\eta)+c T^{2} \tilde{M}^{3} \\
\leq & \left(c \tilde{M}+\tilde{M}^{2}\right) T^{2} \tilde{M}^{2} .
\end{aligned}
$$

Using Definition 9, it can be shown that

$(1-c T)\left(\xi-\bar{\xi}_{T}(\eta)\right) T\left(\hat{d}_{2}+d_{2}\right) \leq 2 T^{2} \tilde{M} \varepsilon$,

and, by bounding $\Lambda^{2}$ by $T^{2} \tilde{M}^{2}(3 \tilde{M}+2)^{2}$ (using the mean value theorem),

$$
\begin{aligned}
& \Delta V_{T} \leq \Delta W_{T}-c T\left(\xi-\bar{\xi}_{T}(\eta)\right)^{2} \\
& \quad+(1-c T)\left(\xi-\bar{\xi}_{T}(\eta)\right)\left(\bar{\xi}_{T}\left(\eta_{0}^{+}\right)-\bar{\xi}_{T}\left(\eta^{+}\right)-T\left(\frac{\partial \bar{\xi}_{T}}{\partial \eta}\left(\eta_{0}^{+}\right)\right)^{\mathrm{T}} \hat{d}_{1}\right) \\
& \quad+T^{2} \tilde{M}\left(\tilde{M}(3 \tilde{M}+2)^{2}+\left(c \tilde{M}+\tilde{M}^{2}\right) \tilde{M}+2 \varepsilon\right) .
\end{aligned}
$$

The term $(1-c T)\left(\xi-\bar{\xi}_{T}(\eta)\right)\left(\bar{\xi}_{T}\left(\eta_{0}^{+}\right)-\bar{\xi}_{T}\left(\eta^{+}\right)\right)$can be written, using the mean value theorem, as

$$
\begin{aligned}
& (1-c T)\left(\xi-\bar{\xi}_{T}(\eta)\right)\left(\bar{\xi}_{T}\left(\eta_{0}^{+}\right)-\bar{\xi}_{T}\left(\eta^{+}\right)\right) \\
& =(1-c T)\left(\xi-\bar{\xi}_{T}(\eta)\right) T\left(\frac{\partial \bar{\xi}_{T}}{\partial \eta}\left(\eta^{\star}\right)\right)^{\mathrm{T}} d_{1},
\end{aligned}
$$

with $\eta^{\star}=\eta+T(f(\eta)+g(\eta) \xi)+T \theta_{2} d_{1}$, and $\theta_{2} \in(0,1)$. Consequently,

$$
\begin{aligned}
\Delta V_{T} & \leq \Delta W_{T}-c T\left(\xi-\bar{\xi}_{T}(\eta)\right)^{2} \\
& +(1-c T) T\left(\xi-\bar{\xi}_{T}(\eta)\right) \\
& \times\left(\left(\frac{\partial \bar{\xi}_{T}}{\partial \eta}\left(\eta^{\star}\right)\right)^{\mathrm{T}} d_{1}-\left(\frac{\partial \bar{\xi}_{T}}{\partial \eta}\left(\eta_{0}^{+}\right)\right)^{\mathrm{T}} \hat{d}_{1}\right) \\
& +T^{2}\left(\tilde{M}(3 \tilde{M}+2)^{2}+\left(c \tilde{M}+\tilde{M}^{2}\right) \tilde{M}+2 \varepsilon\right) \\
\leq & \Delta W_{T}-c T\left(\xi-\bar{\xi}_{T}(\eta)\right)^{2} \\
& +(1-c T) T\left(\xi-\bar{\xi}_{T}(\eta)\right)\left(\left(\frac{\partial \bar{\xi}_{T}}{\partial \eta}\left(\eta^{\star}\right)\right)^{\mathrm{T}} d_{1}\right. \\
& -\left(\frac{\partial \bar{\xi}_{T}}{\partial \eta}\left(\eta_{0}^{+}\right)\right)^{\mathrm{T}} d_{1}+\left(\frac{\partial \bar{\xi}_{T}}{\partial \eta}\left(\eta_{0}^{+}\right)\right)^{\mathrm{T}} d_{1} \\
& \left.-\left(\frac{\partial \bar{\xi}_{T}}{\partial \eta}\left(\eta_{0}^{+}\right)\right)^{\mathrm{T}}\left(\hat{d}_{1}\right)\right) \\
& +T^{2} \tilde{M}\left(\tilde{M}(3 \tilde{M}+2)^{2}+\left(c \tilde{M}+\tilde{M}^{2}\right) \tilde{M}+2 \varepsilon\right) \\
\leq & \Delta W_{T}-c T\left(\xi-\bar{\xi}_{T}(\eta)\right)^{2} \\
& +T^{2}(1-c T)\left|\xi-\bar{\xi}_{T}(\eta)\right| \frac{\partial^{2} \bar{\xi}_{T}}{\partial \eta^{2}}\left(\eta^{\star \star}\right) \mid \theta_{2} \rho_{1}^{2}(\eta)
\end{aligned}
$$

$$
\begin{aligned}
& +(1-c T) T\left(\xi-\bar{\xi}_{T}(\eta)\right)\left[\left(\frac{\partial \bar{\xi}_{T}}{\partial \eta}\left(\eta_{0}^{+}\right)\right)^{\mathrm{T}}\left(d_{1}-\hat{d}_{1}\right)\right] \\
& +T^{2} \tilde{M}\left(\tilde{M}(3 \tilde{M}+2)^{2}+\left(c \tilde{M}+\tilde{M}^{2}\right) \tilde{M}+2 \varepsilon\right),
\end{aligned}
$$

with $\eta^{\star \star}=\eta+T(f(\eta)+g(\eta) \xi)+T \theta_{2} \theta_{3} d_{1}, \theta_{3} \in(0,1)$. Thus, by definition of $\hat{d}_{1}$,

$$
(1-c T) T\left(\xi-\bar{\xi}_{T}(\eta)\right)\left(\frac{\partial \bar{\xi}_{T}}{\partial \eta}\left(\eta_{0}^{+}\right)\right)^{\mathrm{T}}\left(d_{1}-\hat{d}_{1}\right) \leq 2 T^{2} \tilde{M} \varepsilon .
$$

Using Hypothesis 11 and the definition of $\hat{M}$,

$\Delta V_{T} \leq-T \alpha_{3}(|\eta|)-c T\left(\xi-\bar{\xi}_{T}(\eta)\right)^{2}+T \delta$.

From Proposition 1 in Nešić and Teel (2006), there exists $\bar{\alpha}_{3} \in \mathcal{K}_{\infty}$, such that

$\Delta V_{T} \leq-T \bar{\alpha}_{3}(|x|)+T \delta$.

Using the mean value theorem, it can be shown that there exists $\bar{L} \in \mathbb{R}_{>0}$, such that, for all $x, z \in \mathbb{R}^{n+1}$ with $\max \{|x|,|z|\} \leq \Delta$, $\left|V_{T}(x)-V_{T}(z)\right| \leq \bar{L}|x-z|$. Finally,

$$
\begin{aligned}
\left|u_{T}\right| \leq & c\left|\xi-\bar{\xi}_{T}(\eta)\right|+\left|\hat{d}_{2}\right|+\left|\frac{\bar{\xi}_{T}\left(\eta_{0}^{+}\right)-\bar{\xi}_{T}(\eta)}{T}\right| \\
& +\left|\left(\frac{\partial W}{\partial \eta}\left(\bar{\eta}_{0}^{+}\right)\right)^{\mathrm{T}} \| g(\eta)\right|+\left|\left(\frac{\partial \bar{\xi}_{T}}{\partial \eta}\left(\eta_{0}^{+}\right)\right)^{\mathrm{T}}\right|\left|\hat{d}_{1}\right| \\
\leq & 3 \tilde{M}^{2}+\tilde{M}(c+1)=\bar{M} .
\end{aligned}
$$

Consequently, system (13)-(15) is SP-AS, and the same property holds for the exact discretized system (8) and (9), in view of Theorem 3.

Note that if perturbation $d_{1}$ is not vanishing, assuming a slightly modified version of Hypothesis 11, only a $\delta$-regulation property (see Freeman and Kokotović (1993)) can be achieved.

Remark 13. Virtual controller $\bar{\xi}_{T}$ cannot use sat functions parameterized by $T$ (like in Definition 9) since its derivatives are not uniformly bounded w.r.t. $T$.

Remark 14. Like in Section 2, controllers (15) are redesigned compared to the emulation. However, here the sampling $T$ appears not only linearly in the control but also in the definition of the sat functions.

\section{Comments} form

The application of the proposed techniques to systems of the

$$
\begin{aligned}
& \dot{x}_{1}=x_{2}+f_{1}\left(x_{1}\right)+d_{1} \\
& \dot{x}_{2}=x_{3}+f_{2}\left(x_{1}, x_{2}\right)+d_{2} \\
& \vdots \\
& \dot{x}_{n}=u+f_{n}(x)+d_{n},
\end{aligned}
$$

where $x=\left[x_{1}, \ldots, x_{n}\right]^{\mathrm{T}}$ and $u \in \mathbb{R}$, is straightforward for both cases (SP-ISS and SP-AS) by assuming the functions $f_{i}$ to be sufficiently differentiable, and so will be the virtual controllers and their associate Lyapunov functions (Krstić, Kanellakopoulos, \& Kokotović, 1995). Note that for the SP-A stabilization the functions $d_{i}, i \in\{1, \ldots, n-1\}$, have to be vanishing. On the other hand, the extension of these results to higher order approximations of the exact discretized model of (8) and (9) will require additional knowledge about the perturbations terms and their successive derivatives. 

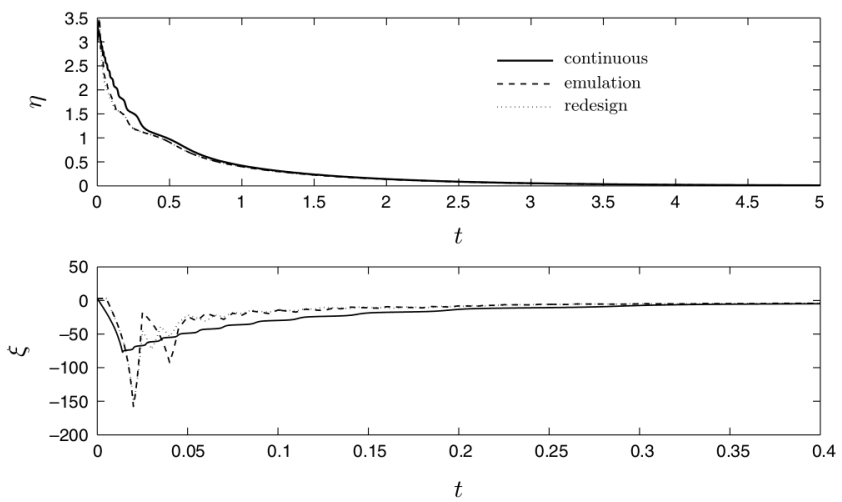

Fig. 1. Simulation results for $\eta(0)=\xi(0)=3$ and $T=0.005$.
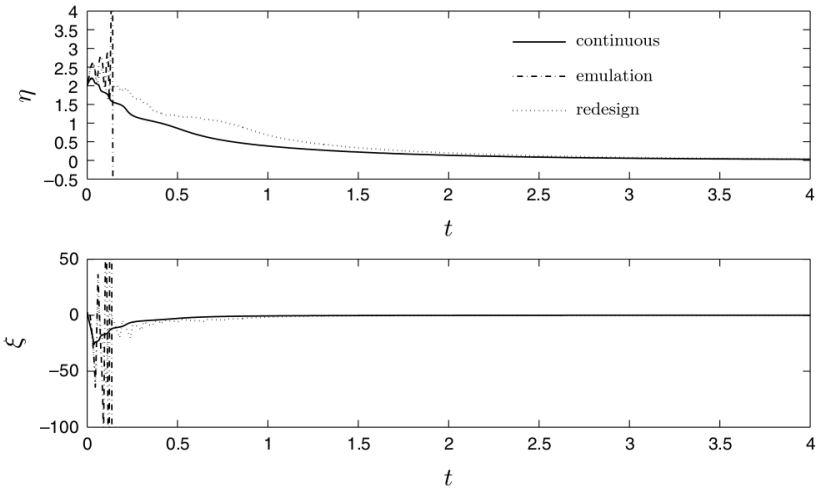

Fig. 2. Simulation results for $\eta(0)=\xi(0)=2$ and $T=0.015$.

\section{Illustrative example}

Consider the two-dimensional nonlinear system

$\dot{\eta}=\eta^{2}+\xi+d_{1}(x)$

$\dot{\xi}=u+d_{2}(x)$,

where $d_{1}: x \longmapsto\left(1+\sin \left(x_{2}\right)\right) x_{1}^{3}$ is bounded by $\rho_{1}: x \longmapsto 2\left|x_{1}\right|^{3}$ and $d_{2}: x \longmapsto 1+\cos \left(x_{1}\right) x_{2}^{2}$ by $\rho_{2}: x \longmapsto 1+x_{2}^{2}$. Taking $\bar{\xi}=$ $-\eta-\eta^{2}-2 \eta^{3}$, Hypotheses 7 and 11 are satisfied; thus Theorem 12 applies. A controller of the form (15) has been designed with $c=1$ and $\varepsilon=0.01$. Some simulations have been performed in order to compare controller (15) with the emulation of a continuoustime one of the 'hard'-type, like in Freeman and Kokotović (1993). The following simulation parameters have been taken: $T=0.005$, $\eta(0)=\xi(0)=3$. Fig. 1 shows that both controllers ensure the convergence of the states to a neighbourhood of the origin, but faster with controller (15). Choosing the sampling period to be $T=0.015$ (and with $\eta(0)=\xi(0)=2$ ), see Fig. 2, it can be seen that the emulation cannot stabilize the system contrary to (15). Thus, the redesigned controller has also enlarged the domain of attraction.

\section{Conclusion}

The SP-ISS and SP-AS stabilization for the Euler approximate model of perturbed sampled-data strict-feedback systems has been addressed. Simulations show that the obtained controllers may improve some system performances compared to the use of the emulation.

\section{Acknowledgement}

The authors are very grateful to Prof. Dragan Nešić who suggested addressing this issue.

\section{References}

Barbot, J. P., Djemai, M., Monaco, S., \& Normand-Cyrot, D. (1996). Analysis and control of nonlinear singularly perturbed systems under sampling. In Leondes (Ed.)., Control and dynamic systems, advances in theory and applications (pp. 203-246). Academic Press Inc.

Burlion, L. (2007). Contribution à l'analyse et à la commande de systèmes non linéaires à commande échantillonnée. Ph.D. thesis. Université Paris-Sud [in French].

Freeman, R. A., \& Kokotović, P. V. (1993). Design of softer robust nonlinear control laws. Automatica, 29, 1425-1437.

Grüne, L., Worthmann, K., \& Nešić, D. (2007). Continuous-time controller redesign for digital implementation: A trajectory based approach. Automatica, 44(1), 225-232.

Kellett, C. M., Shim, H., \& Teel, A. R. (2004). Further results on robustness of (possibly discontinuous) sample and hold feedback. IEEE Transactions on Automatic Control, 49, 1081-1089.

Krstić, M., Kanellakopoulos, I., \& Kokotović, P. V. (1995). Nonlinear and adaptive control design. New York, USA: John Wiley and Sons, Inc.

Laila, D. S., \& Astolfi, A. (2005). Input-to-state stability for discrete-time timevarying systems with applications to robust stabilization of systems in power form. Automatica, 41, 1891-1903.

Laila, D. S., \& Nešić, D. (2002a). A note on input-to-state stabilization for nonlinear sampled-data systems. IEEE Transactions on Automatic Control, 47, 1153-1158.

Laila, D. S., \& Nešić, D. (2002b). Open and closed loop dissipation inequalities under sampling and controller emulation. European Journal of Control, 18, 109-125.

Laila, D. S., \& Nešić, D. (2003). Changing supply rates for input-output to state stable discrete-time nonlinear systems with applications. Automatica, 39, 821-835.

Monaco, S., \& Normand-Cyrot, D. (2001). Issues on nonlinear digital systems. European Journal of Control, 7, 160-178.

Nešić, D., \& Grüne, L. (2005). Lyapunov based continuous-time nonlinear controller redesign for sampled-data implementation. Automatica, 41, 1143-1156.

Nešić, D., \& Teel, A. R. (2004). A framework for stabilization of nonlinear sampleddata systems based on their approximate discrete-time. IEEE Transactions on Automatic Control, 49, 1103-1122.

Nešić, D., \& Teel, A. R. (2006). Stabilization of sampled-data nonlinear systems via backstepping on their Euler approximate model. Automatica, 42, 1801-1808.

Nešić, D., Teel, A. R., \& Sontag, E. D. (1999). Formulas relating $\mathcal{K} \mathcal{L}$ stability estimates of discrete-time and sampled-data nonlinear systems. Systems $\mathcal{E}$ Control Letters, 38(1), 49-60.

Postoyan, R., Ahmed-Ali, T., Burlion, L., \& Lamnabhi-Lagarrigue, F. (2008). On the Lyapunov-based adaptive control redesign for a class of nonlinear sampled-data systems. Automatica, 44(8), 2099-2107. 